\title{
Design of Energy Efficient Four Finger Robotic Hand
}

\author{
Bhivraj Suthar*, Nidhi Sindhu** \\ * Departement of Mechanical Engineering, Indian Institute of Technology Delhi, India \\ ** Departement of Mechanical Engineering, Deen Bandhu Chhotu Ram University of Science and Technology, Haryana
}

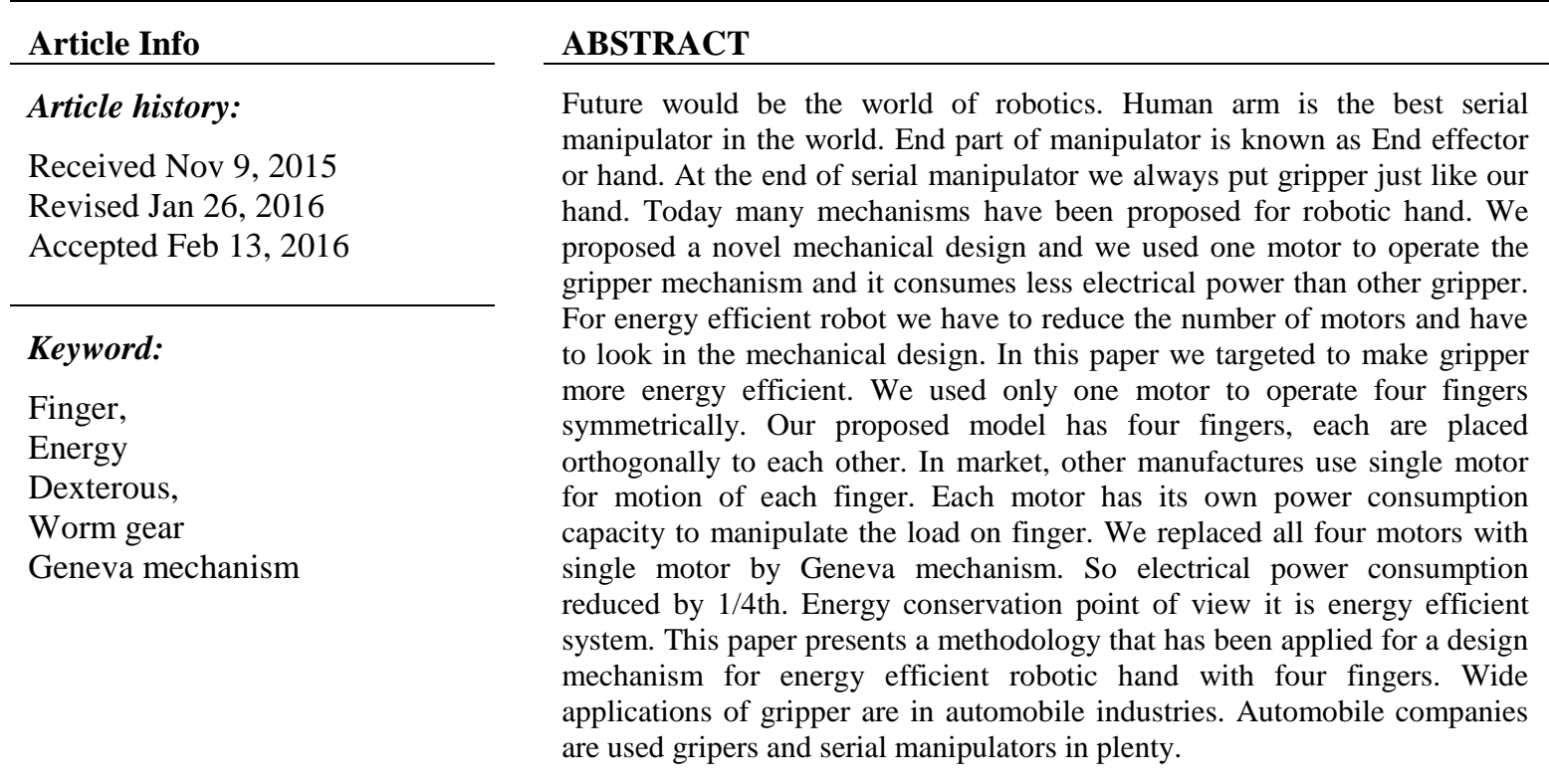

Copyright $(2016$ Institute of Advanced Engineering and Science. All rights reserved.

\section{Corresponding Author:}

Bhivraj Suthar,

Departement of Mechanical Engineering,

Indian Institute of Technology Delhi, India

Email: bhivraj.iitd@gmail.com

\section{INTRODUCTION}

One challenge in automating the item picking is that the objects to be dealt with can be of any shape, so tailoring the gripper to a specific object is impossible, for the variety of the products stored in the distribution center depends on the market and changes over time. If the object to be dealt with is box-like, the vacuum gripper is probably a good solution already. However, in the distribution center sometimes the object does contain no flat surfaces. To handle those objects, the dexterous robotic grippers which are able to handle diverse objects, like the Robonaut Hand [2], or the Gold finger [3], are possible solutions. However this kind of grippers is expensive because a considerable amount of actuators and sensors are involved and a sophisticated control is needed.

The existing under actuated grippers, however, are overdesigned for having the adaptability. For 1 DOA, the gripper of 2 DOF can already exhibit the adaptability, while among the grippers listed in the literature [5] it is found that the DOF they own are all more than enough. The benefit of having many exceeding DOF is that the under actuated gripper might be able to adapt to an object more thoroughly, but there are two reasons for better keeping the degrees of under actuation small. Firstly, the complexity of the analysis of the under actuated grippers dramatically increases with respect to increase of the DOU because of the passivity of the under actuated fingers [6]. However, a careful analysis on the under actuated gripper in the design phase is indispensable because the success of the under actuated grasping is not always 
guaranteed. Secondly, if the gripper containing a small number of degrees of under actuation is able to do the item picking well, a more complex under actuated gripper will be unnecessary.

To have a very simplified under actuated gripper, it is proposed that the gripper is formed by four finger in opposition to one under actuated finger which has two phalanges. the target object is laid in the tote which has an upward opening, the item picking should be conducted from the top of the object. In this situation the only moving finger, the under actuated finger, might miss the object and cannot pick it up according to the geometry. To prevent it, therefore in addition to the concept of fixed finger, in a free grasp the fingertip of the under actuated finger is intended to move along the plane surface where the object is lying, so that the most distal phalanx is always able to reach and shovel the object up by going under it.

Objective and Approach The objective of this paper is to assess the workability and the performance of the gripper with the proposed concepts on the item picking. To approach the objective, a gripper is to be designed, built, and tested with the consideration of the following two specific tasks:

(a) The gripper has to pick a cylindrical object initially lying on the ground only one target object is considered in one grasp action, and the ground is meant to be a plane surface. The cylindrical object is chosen because it has the simplest form out of the non-box-like items; it can be modeled by only one variable in a planar case. Initially, the cylindrical object is at rest with an orientation that its central axis is parallel to ground.

(b) The gripper has to retain the grasp on the object when the grasp is undergoing a lifting acceleration the grasp is disturbed in the item picking when the gripper together with the grasped object is in transportation. The disturbance introduced by a lifting transportation seems to be the worst case scenario because the opening of the gripper is downward.

\section{MECHANICAL DESIGN OF ROBOTIC HAND}

\subsection{Design of Four Finger}

A mechanical model, formulated based on the observation of our hand motion. It has generally four segments of body and perform the gripping with the help of four fingers. The entire body of the robot and the grippers can be modeled as inchworm mechanism and worm gear. When that robotic hand pick any object than action and reaction forces appear on each finger as shown in figure 1 below.

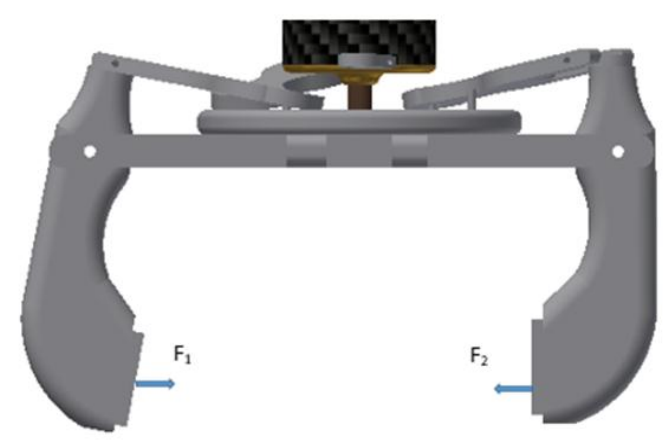

Figure 1. Forces on two finger in robotic hand

The links would have to rotate about the central joint where the servo is connected in order to close the fore gripper. Not only that, we would need to control the extension and contraction of the trunk/segment also by using same DC motor.

\subsection{Design of Grippers}

The design of the gripper is an essential part of the robotic hand gripper. During the picking, finger has to bear the entire weight of the pay load or object. This, is the main consideration for the design of the gripper. The gripping mechanism operated by the same DC motor. The four fingers of gripper, are connected with one DC motor and few links as shown in figure 3.

\subsection{Complete Design}

The design of the robotic hand based on inch worm mechanism and four finger completes the mechanical design. Figure 2, below, shows the complete design with the gripper with forces. 


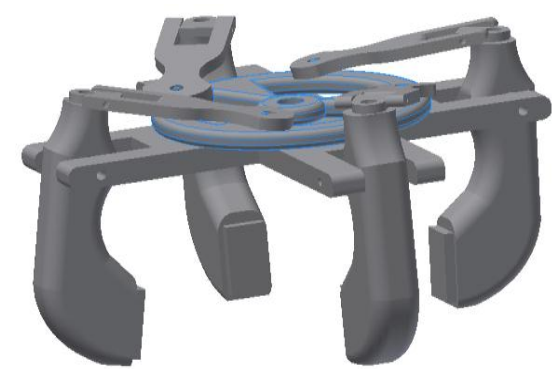

Figure 2. Complete CAD design of four finger robotic hand

\section{GRIPER ANALYSIS}

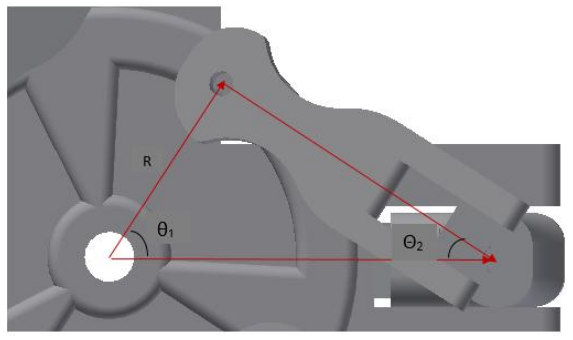

Figure 3. The Kinematic analysis for verifying the relation between the robot size and rope curvature

$$
\tan \theta_{3}=\frac{R_{2} \sin \theta_{2}}{X-R_{2} \cos \theta_{2}}
$$

Assuming $\mathrm{X}$ is constant and differentiating with respect to time

$$
\omega_{3}=\omega_{2}\left[\frac{\frac{X}{R_{2}} \cos \theta_{2}-1}{1+\left(\frac{X}{R_{2}}\right)^{2}-2\left(\frac{X}{R_{2}} \cos \theta_{2}\right)}\right]
$$

Differentiating with respect to time again

$$
\left.\left.\alpha_{3}=\omega_{2}^{2}\left[\frac{\frac{X}{R_{2}} \sin \theta_{2}\left\{1-\left(\frac{X}{R_{2}}\right)^{2}\right\}}{\left[1+\left(\frac{X}{R_{2}}\right)^{2}-2\left\{\left(\frac{X}{R_{2}} \operatorname{Cos} \theta_{2}\right)\right\}\right.}\right]\right]^{2}\right]
$$

Where $\alpha$ is acceleration of finger.

Each finger has same acceleration. But we used only one motor to produce identical acceleration at each finger of robotic hand. Our proposed robotic hand is symmetrical about center axis of pam of hand. Shape is also responsible for effective pick and place operation.

Although in market various type of robotic hand available but all have more than one motor to operate all fingers. Above equation shows that each force at each finger is directly proportional to the acceleration. We placed DC motor connect to disc with worm gear arrangement. The best advantage of worm 
gear is self-locking. This novel design does not need any locking mechanism after picking operation because this worm gear provide self-locking.

\subsection{Robot Gripper Sizing}

The four finger move by one DC motor and figure 4 shows the acting forces in vector form. Both angles will change by the DC motor, during this motion worm gear mechanism play a main role. In above section we setup a relation between both angles. The maximum opening of the gripper is $10 \mathrm{~cm}$ and minimum opening is $2 \mathrm{~cm}$. so pay load laying between this ranges for effective gripping.

\section{PROTOTYPE DESIGN}

\subsection{Proto Type Inch Worm Mechanism Testing}

The robot's bodies are made of wooden to reduce the weight, and the crank and joints are made. It has one motor and responsible for all four finger motion shown in figure 5.

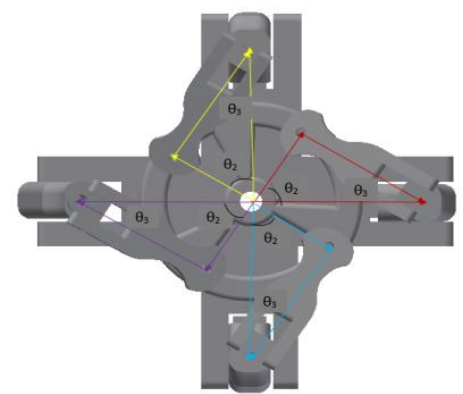

Figure 4. Four fingers with vector forces

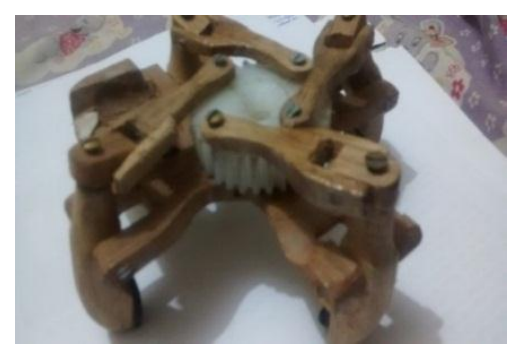

Figure 5. Prototype of four finger robotic hand

\subsection{Specification of DC motor}

Table (a) Electrical specification

\begin{tabular}{|c|c|c|c|}
\hline \multicolumn{2}{|l|}{ VOLTAGE } & \multicolumn{2}{|c|}{ NO LOAD } \\
\hline OPERATING & $\begin{array}{l}\text { NOMI- } \\
\text { NAL } \\
\end{array}$ & SPEED & CURRENT \\
\hline RANGE & V & $\mathrm{rpm}$ & A \\
\hline $3.0-18$ & 12 & 2700 & 0.020 \\
\hline
\end{tabular}

Table (b) Electrical specification

\begin{tabular}{|l|c|c|c|c|c|c|l|}
\hline \multicolumn{7}{|c|}{ AT MAXIMUM EFFICIENCY } & \multicolumn{2}{|c|}{ STALL } \\
\hline SPEED & $\begin{array}{l}\text { CUR- } \\
\text { RENT }\end{array}$ & \multicolumn{2}{|l|}{ TORQUE } & OUTPUT & TORQUE & \multicolumn{2}{c|}{$\begin{array}{l}\text { CUR- } \\
\text { RENT }\end{array}$} \\
\hline rpm & A & mN.m & g.cm & w & mN.m & g.cm & A \\
\hline 2200 & 0.080 & 0.98 & 10.0 & 0.23 & 5.88 & 60 & 0.5 \\
\hline
\end{tabular}

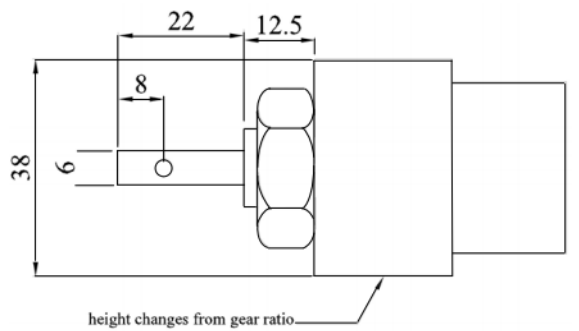

Figure 6. Mechanical dimension of DC motor 


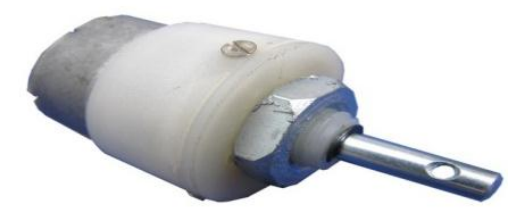

Figure 7. 12V DC motor used in four finger robotic hand

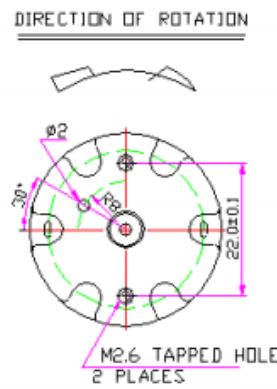

UNIT: mm

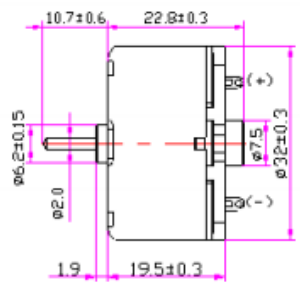

VEIGHT:45g(Apprax)

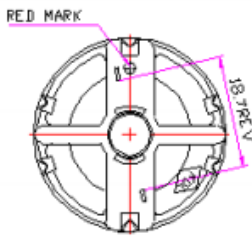

(4)

Figure 8. Mechanical drawing of DC motor

\section{FUTURE WORK AND CONCLUSION}

In this paper inchworm and worm mechanism based four finger gripper has been made and tested for the pick-place operation in CAD as well as prototype also. Rotation of disc has attached with four fingers via various links which has revolute joint only. This design scheme reduces the consumption by one fourth.

The inchworm and worm motion was studied in detail. CAD model, prototype model and a kinematic model were made. In kinematics analysis setup a mathematical relation for gripper. We calculated acceleration of each finger. Mathematical expression was setup.

A control system for whole gripper will design in future such that the gripper and the segments when operate in coordination would simulate the pick \& place operation. If the hinge joints in the segments of the robot were replaced by ball-and-socket joints, then the robot would have much more flexibility and smooth operation.

\section{ACKNOWLEDGMENT}

Thanks for Mr. Balkishan Suthar and are due to all the people and things that have directly or indirectly influenced me and helped me during the design and manufacturing of four finger robotic hand.

\section{PATENT}

Bhivraj Suthar and Nidhi Sindhu "Design of four finger hand" Provisionally patent filed, Application no- 409/DEL/2015.

\section{REFERENCES}

[1] Vanderlande Industries: Distribution, http://www.vanderlande.com/web/products-and -solutions.htm, 2010.

[2] M. A. Diftler and R. O. Ambrose, "Robonaut: A Robotic Astronaut Assistant," Proceedings of the 6th International Symposium on Artificial Intelligence and Robotics and Automation in Space, Canadian Space Agency, 2001.

[3] A. M. Ramos, I. A. Gravagne, and I. D. Walker, "Goldfinger: A Non-Anthropomorphic, Dextrous Robot Hand," Proceedings of the 1999 IEEE International Conference on Robotics and Automation, pp. 913-919, 1999.

[4] Y. Chang, "Underactuated Grippers for Distribution Centers: Evaluation and Recommendation," Literature Report, Delft University of Technology, 2009.

[5] L. Birglen, "An Introduction to the Analysis of Linkage-driven Compliant Underactuated Fingers," Proceedings of ASME 2006 International Design Engineering Technical Conferences, DETC2006-99048, pp. 1-9, 2006.

[6] V. Bégoc, S. Krut, E. Dombre, C. Durand and F. Pierrot, "Mechanical Design of a New Pneumatically Driven Underactuated Hand," Proceedings of the 2007 IEEE International Conference on Robotics and Automation, pp. 927-933, 2007 